\title{
Analysis of ultra-triathlon performances
}

This article was published in the following Dove Press journal:

Open Access Journal of Sports Medicine

24 August 2011

Number of times this article has been viewed

Romuald Lepers'

Beat Knechtle $e^{2,3}$

Patrizia Knechtle ${ }^{2}$

Thomas Rosemann ${ }^{2}$

'INSERM U887, Faculty of Sport Sciences, University of Burgundy, Dijon, France; ${ }^{2}$ Institute of General Practice and Health Services Research, University of Zurich, Switzerland; ${ }^{3}$ Gesundheitszentrum St Gallen, St Gallen, Switzerland

Correspondence: Romuald Lepers INSERM U887, Université de Bourgogne, BP 27877, 21078 Dijon Cedex, France Tel +33380396760

$\mathrm{Fax}+33380396702$

Email romuald.lepers@u-bourgogne.fr
Abstract: Despite increased interest in ultra-endurance events, little research has examined ultra-triathlon performance. The aims of this study were: (i) to compare swimming, cycling, running, and overall performances in three ultra-distance triathlons, double Ironman distance triathlon (2IMT) (7.6 km swimming, $360 \mathrm{~km}$ cycling, and $84.4 \mathrm{~km}$ running), triple Ironman distance triathlon (3IMT) $(11.4 \mathrm{~km}, 540 \mathrm{~km}$, and $126.6 \mathrm{~km})$, and deca Ironman distance triathlon (10IMT) $(38 \mathrm{~km}, 1800 \mathrm{~km}$, and $420 \mathrm{~km})$ and (ii) to examine the relationships between the 2IMT, 3IMT, and 10IMT performances to create predicted equations of the 10IMT performances. Race results from 1985 through 2009 were examined to identify triathletes who performed the three considered ultra-distances. In total, 73 triathletes (68 men and 5 women) were identified. The contribution of swimming to overall ultra-triathlon performance was lower than for cycling and running. Running performance was more important to overall performance for 2IMT and 3IMT compared with 10IMT. The 2IMT and 3IMT performances were significantly correlated with 10IMT performances for swimming and cycling, but not for running. 10IMT total time performance might be predicted by the following equation: 10IMT race time $($ minutes $)=5885+3.69 \times 3$ IMT race time (minutes). This analysis of human performance during ultra-distance triathlons represents a unique data set in the field of ultra-endurance events. Additional studies are required to determine the physiological and psychological factors associated with ultra-triathlon performance.

Keywords: triathlon, swimming, cycling, running, ultra-endurance

\section{Introduction}

Despite increased interest in ultra-endurance events, little research has examined ultratriathlon performance. The ultra-triathlon involves swimming, cycling, and running in immediate succession over various distances, such as double Ironman distance triathlon (2IMT) (7.6 km swimming, $360 \mathrm{~km}$ cycling, and $84.4 \mathrm{~km}$ running), triple Ironman distance triathlon (3IMT) $(11.4 \mathrm{~km}, 540 \mathrm{~km}$, and $126.6 \mathrm{~km})$, and deca Ironman distance triathlon (10IMT) $(38 \mathrm{~km}, 1800 \mathrm{~km}$, and $420 \mathrm{~km})$. Ultra-triathletes compete for fastest overall course completion time, including transitions between the individual swim, bike, and run components, and recovery periods for nutrition and sleep. The swimming part takes place in open-water or in a swimming pool. The cycling and running courses usually consist of several loops that are closed to automobile traffic and illuminated during the night. All triathletes have their own support crew to provide nutrition and changes of clothing and equipment.

Participation in ultra-triathlons has increased during the last 25 years. ${ }^{1}$ From 1985 to 2009 , the total number of 2IMT, 3IMT, and 10IMT events around the world were 
85,48 , and 10 , respectively. During the same period, the total number of triathletes finishing 2IMT, 3IMT, and 10IMT were 1333, 796, and 127, respectively. ${ }^{1}$ Performance in Ironman distance triathlons $(3.8 \mathrm{~km}$ swimming, $180 \mathrm{~km}$ cycling, and $42.2 \mathrm{~km}$ running) has already been well analyzed, ${ }^{2-4}$ but performance in ultra-triathlons has received little attention. ${ }^{5-7}$ For example, the relative contributions of physiological, psychological, anthropometric, and training parameters to ultratriathlon performance remain unclear. It has been shown that anthropometric factors such as body mass index, body fat, and skeletal mass have no influence on 3IMT performance. ${ }^{8}$ Similarly, during a different ultra-endurance event consisting of performing ten Ironman distance triathlons over 10 consecutive days, there was no correlation between anthropometric parameters and race performance. ${ }^{9}$

The relative contributions of each discipline to overall performances in ultra-triathlons might be of interest in the success of ultra-triathlons. For example, Knechtle and Kohler $^{8}$ showed that 3IMT performance was associated with cycling and running performance, but not with swimming performance. However, this study was conducted on 17 male finishers only. To our knowledge, swimming, cycling, and running performance times at 10IMT have never been described in the literature. In addition, it would be interesting to examine the relationships between 2IMT, 3IMT, and 10IMT performances in order to predict 10IMT performance based on 2IMT and 3IMT performances.

The aims of this study were therefore: (i) to compare swimming, cycling, running, and overall performances in three ultra-distance triathlons, 2IMT, 3IMT, and 10IMT, respectively, and (ii) to examine the relationships between the 2IMT, 3IMT, and 10IMT performances to create predicted equations of 10IMT performances.

\section{Materials and methods Subjects}

Approval for the project was obtained from the Burgundy University Committee on Human Research. This study involved the analysis of publicly available data so consent was waived.

\section{Data analysis}

Results for ultra-triathlons throughout the world from 1985 through 2009 were retrieved from publicly available sources including different individual race websites (http://www. multisport.com.mx; http://www.triathlonneulengbach.at; http://www.triathlonlensahn.de), international ultra-triathlon association (www.iutasport.com), and from direct contact with race directors. We identified all triathletes who finished at least one ultra-triathlon in each of three distances, 2IMT, 3IMT, and 10IMT during a 5-year period. When a triathlete performed more than one race over an ultra-distance, only the personal best performance was considered. A total of 73 triathletes ( 68 male and 5 female) were identified as finishing at least one triathlon of the three ultra-distances. The data extracted included name, sex, age, nationality, name and year of the race, swimming, cycling, running, and total times.

\section{Statistical analysis}

Data are reported as mean $\pm \mathrm{SD}$ within the text and the tables. The relationships between swimming, cycling, running, and total times of the three ultra-distances were analyzed using linear regression analysis. Stepwise multiple regression analysis was then used to determine the best variables correlated with the 10IMT performance. One-way ANOVA was used to compare the speed in the three disciplines across the different ultra-distances, and the speed loss between the different ultra-distances (Statsoft, v 6.1; Statistica, Tulsa, OK). A significance level of $P<0.05$ was used to identify statistical significance.

\section{Results}

The total number of ultra-triathletes per continent is shown in Figure 1. Seventy-nine percent of the ultra-triathletes in the present study were from Europe. The European and North American nations most represented were: France $(n=10)$, Great Britain $(n=10)$, Germany $(n=9)$, Austria $(n=6)$, Italy $(n=5)$, US $(n=7)$, and Canada $(n=4)$.

Age, swimming, cycling, running, and total time performances of the male and female ultra-triathletes are presented in Table 1. The relative swim contributions to the total time were $8.9 \% \pm 1.9 \%, 8.8 \% \pm 1.3 \%$, and

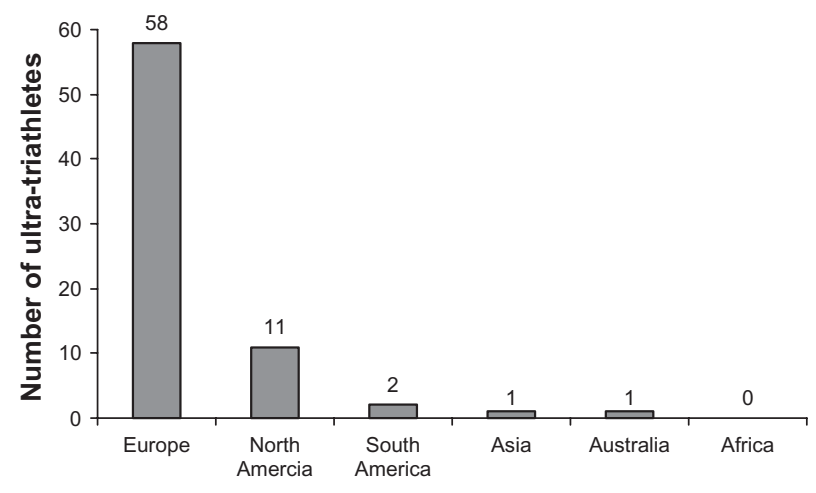

Figure I Total number of ultra-triathletes per continent included in the present study $(n=73)$. 
Table I Age, swimming, cycling, running, and total performance times for ultra-triathletes who performed double (2IMT), triple (3IMT), and deca (IOIMT) Ironman distance triathlons

\begin{tabular}{|c|c|c|c|}
\hline & $\begin{array}{l}21 M T \\
(7.6-360-84.4 \mathrm{~km})\end{array}$ & $\begin{array}{l}3 \mathrm{IMT} \\
(\mathrm{II} .4-540-126.6 \mathrm{~km})\end{array}$ & $\begin{array}{l}\text { I0IMT } \\
(38-1800-420 \mathrm{~km})\end{array}$ \\
\hline \multicolumn{4}{|l|}{ Male $(n=68)$} \\
\hline Age (yrs) & $37.2 \pm 8.0$ & $37.5 \pm 8.1$ & $38.6 \pm 8.3$ \\
\hline Swim (min) & $140 \pm 29$ & $242 \pm 43$ & $1097 \pm 256$ \\
\hline Cycle (min) & $795 \pm 104$ & $1379 \pm 242$ & $7895 \pm 1449$ \\
\hline Run (min) & $642 \pm 122$ & $1164 \pm 282$ & $7091 \pm 1642$ \\
\hline Total (min) & $1579 \pm 203$ & $2786 \pm 459$ & $16108 \pm 2715$ \\
\hline Total (\% winner time) & $121 \pm 14$ & $|30 \pm 2|$ & $130 \pm 21$ \\
\hline \multicolumn{4}{|l|}{ Female $(n=5)$} \\
\hline Age (yrs) & $37.8 \pm 5.8$ & $38.6 \pm 6.5$ & $40.0 \pm 7.2$ \\
\hline Swim (min) & $142 \pm 37$ & $268 \pm 13$ & $1203 \pm 167$ \\
\hline Cycle (min) & $855 \pm 84$ & $1508 \pm 185$ & $9737 \pm 2024$ \\
\hline Run (min) & $758 \pm 128$ & $1309 \pm 174$ & $7249 \pm 659$ \\
\hline Total (min) & $1760 \pm 213$ & $3085 \pm 343$ & $18190 \pm 2112$ \\
\hline Total (\% winner time) & $138 \pm 19$ & $138 \pm 2 \mid$ & $143 \pm 16$ \\
\hline
\end{tabular}

Notes: When triathletes performed several triathlons in the same ultra-distance, only the best performance was considered. Values are means \pm SD.

$6.9 \% \pm 1.6 \%$ for $2 \mathrm{IMT}$, 3IMT, and 10IMT, respectively. The relative cycle contributions to the total time were $50.3 \% \pm 3.3 \%, 49.6 \% \pm 4.8 \%$, and $49.4 \% \pm 5.1 \%$ for 2IMT, 3IMT, and 10IMT, respectively. The relative run contributions to the total time were $40.6 \% \pm 4.1 \%$, $41.5 \% \pm 5.1 \%$, and $43.5 \% \pm 5.5 \%$ for 2IMT, 3IMT, and 10IMT, respectively.

Averaged swimming, cycling, and running speeds and corresponding speed losses between the three ultra-triathlons are presented in Table 2. The speed in the three modes of locomotion significantly $(P<0.001)$ decreased when the ultra-distance increased. The speed losses between 2IMT and 3IMT did not differ among the three disciplines. The speed losses between 2IMT and 10IMT and between 3IMT and 10IMT were significantly smaller $(P<0.01)$ in swimming than in cycling and running.

Swimming, cycling, and running times were significantly correlated $(P<0.001)$ with total time for the three ultradistances except swimming time and total time for 2IMT (see Table 3).
The correlations between 2IMT and 10IMT performances and between 3IMT and 10IMT performances are shown in Figures 2 and 3, respectively. 2IMT and 3IMT total times were significantly correlated with 10IMT total time. 2IMT and 3IMT swimming times were significantly correlated with 10IMT swimming time, but to a greater extent for 3IMT. 2IMT and 3IMT cycling times were significantly correlated with 10IMT cycling time. In contrast, for running there was neither significant correlation between 2IMT and 10IMT performances nor between 3IMT and 10IMT performances. According to stepwise multiple regressions, 10IMT time performances for swimming, cycling, and total event might be predicted by the following equations: swimming $\left(r^{2}=0.36\right)$ :10IMT time $($ minutes $)=236+3.57 \times 3 \mathrm{IMT}$ time $($ minutes $)$; cycling $\left(r^{2}=0.41\right): 10 \mathrm{IMT}$ time (minutes) $=382+5.90 \times 2 \mathrm{IMT}$ time (minutes) $+2.11 \times 3$ IMT time (minutes); total event $\left(r^{2}=0.39\right): 10 \mathrm{IMT}$ time $($ minutes $)=5885+3.69 \times 3 \mathrm{IMT}$ time (minutes). No prediction was possible for running because there was no significant correlation between 2IMT and 3IMT performances and 10IMT performance.

Table 2 Swimming, cycling, and running averaged speed for double (2IMT), triple (3IMT), and deca (I OIMT) Ironman distance triathlons, and corresponding speed loss between the three ultra-distances

\begin{tabular}{lrrrrrr}
\hline $\begin{array}{l}\text { Speed } \\
\left(\mathbf{k m} \cdot \mathbf{h}^{-1}\right)\end{array}$ & 2IMT & 3IMT & IOIMT & $\begin{array}{l}\text { Speed loss (\%) } \\
\text { IOIMT vs 2IMT }\end{array}$ & $\begin{array}{l}\text { Speed loss (\%) } \\
\text { I0IMT vs 3IMT }\end{array}$ & $\begin{array}{l}\text { Speed loss (\%) } \\
\text { 3IMT vs 2IMT }\end{array}$ \\
\hline Swimming & $3.4 \pm 0.8$ & $2.9 \pm 0.5$ & $2.2 \pm 0.5$ & $-33.8 \pm 16.6$ & $-24.5 \pm 13.4$ & $-11.8 \pm 18.4$ \\
Cycling & $27.5 \pm 3.4$ & $24.0 \pm 4.1$ & $14.0 \pm 2.7$ & $-49.1 \pm 7.8 * *$ & $-41.3 \pm 10.0 * *$ & $-12.2 \pm 13.2$ \\
Running & $8.0 \pm 1.6$ & $6.8 \pm 1.6$ & $3.7 \pm 0.9$ & $-52.5 \pm 12.1 * *$ & $-42.9 \pm 17.8 * *$ & $-15.3 \pm 13.1$ \\
\hline
\end{tabular}

Notes: The speed in the three disciplines significantly decreased $(P<0.00 I)$ when the ultra-distance increased. $* * P<0.01$, speed loss significantly different from swimming. Values are means $\pm S D$. 
Table 3 Squared correlation coefficients between the time per discipline and the total race time for double (2IMT), triple (3IMT), and deca (IOIMT) Ironman distance triathlons $(n=73)$

\begin{tabular}{llll}
\hline & $\begin{array}{l}\text { Swimming } \\
\text { time }\end{array}$ & $\begin{array}{l}\text { Cycling } \\
\text { time }\end{array}$ & $\begin{array}{l}\text { Running } \\
\text { time }\end{array}$ \\
\hline IOIMT total time & 0.17 & 0.69 & 0.46 \\
3IMT total time & 0.30 & 0.64 & 0.73 \\
2IMT total time & 0.05 & 0.76 & 0.76 \\
\hline
\end{tabular}

Notes: All correlations were significant at $P<0.001$, except total time and swimming time for 2IMT $(P=0.053)$.

\section{Discussion}

The aims of the present study were to compare swimming, cycling, running, and overall performances in different ultra-distance triathlons and to examine the relationships between the 2IMT, 3IMT, and 10IMT performances to create predicted equations of 10IMT performances. This study is the first to analyze performances of ultra-distance triathlons from 2IMT to 10IMT which is currently the longest distance in ultra-triathlon. Analysis of the performances of the same athletes enhances the significance of the comparison between the different ultra-distances. Finishing a 10IMT represents a physical and mental challenge that few ultra-triathletes had achieved (less than 120 finishes in the world ${ }^{4}$ ) and the present results obtained for 73 subjects represents a unique data set in the field of ultra-endurance events.
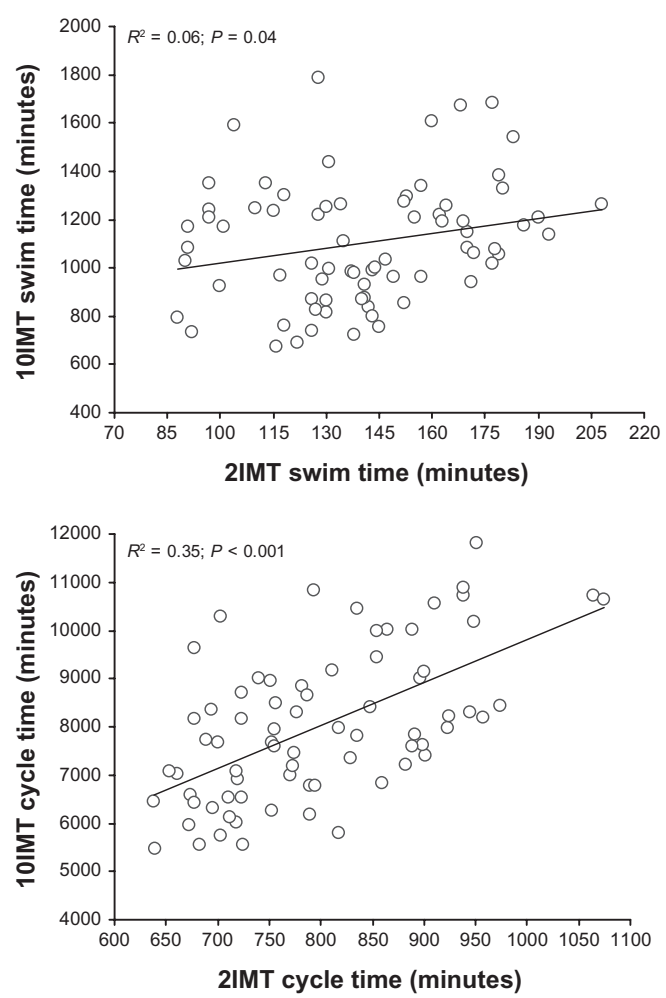

Our results suggest that ultra-triathletes perform their personal best at approximately 37 to 40 years of age whatever the ultra-triathlon distance. For shorter ultra-distances such as the Ironman triathlon, Lepers et $\mathrm{al}^{2}$ found that performance remained stable until approximately 40 years of age. Similar findings were observed for ultra-marathons such as 160-km running competitions ${ }^{10,11}$ suggesting that in ultra-distance events, athletes can still achieve maximal performances until 40 years of age.

When the distance of ultra-triathlon increased, the speed in the three disciplines significantly decreased. Swimming, cycling, and running speeds decreased by $12 \%$ to $15 \%$ when distance increased from 2IMT to 3IMT, but the speed losses were much greater when distance increased from 2IMT/3IMT to 10IMT. Compared with 2IMT performance, 10IMT performance was less altered in swimming compared with running and cycling. Cycling and running speeds were approximately divided by two between 2IMT and 10IMT. The shorter duration of swimming compared with cycling and running, and the fact that swimming is the first discipline, may explain this result.

Swimming time performance is much less closely related to total time performance than cycling and running time performance whatever the ultra-distance triathlon. This result can be explained by the shortest duration of the swimming
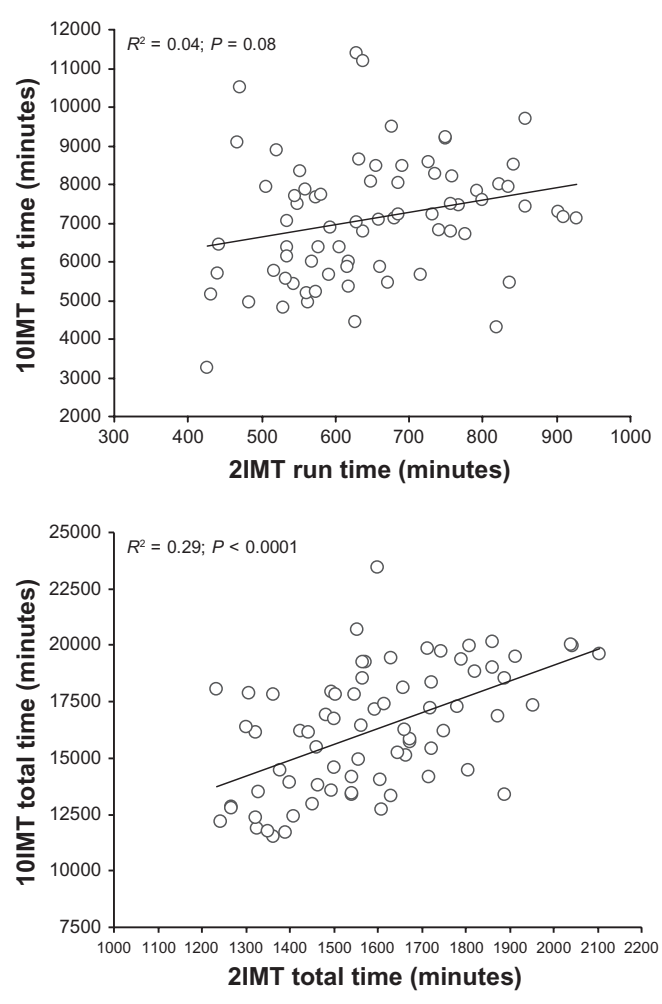

Figure 2 Relationships between double (2IMT) and deca (IOIMT) Ironman distance triathlons performance for swimming, cycling, running, and total event. 

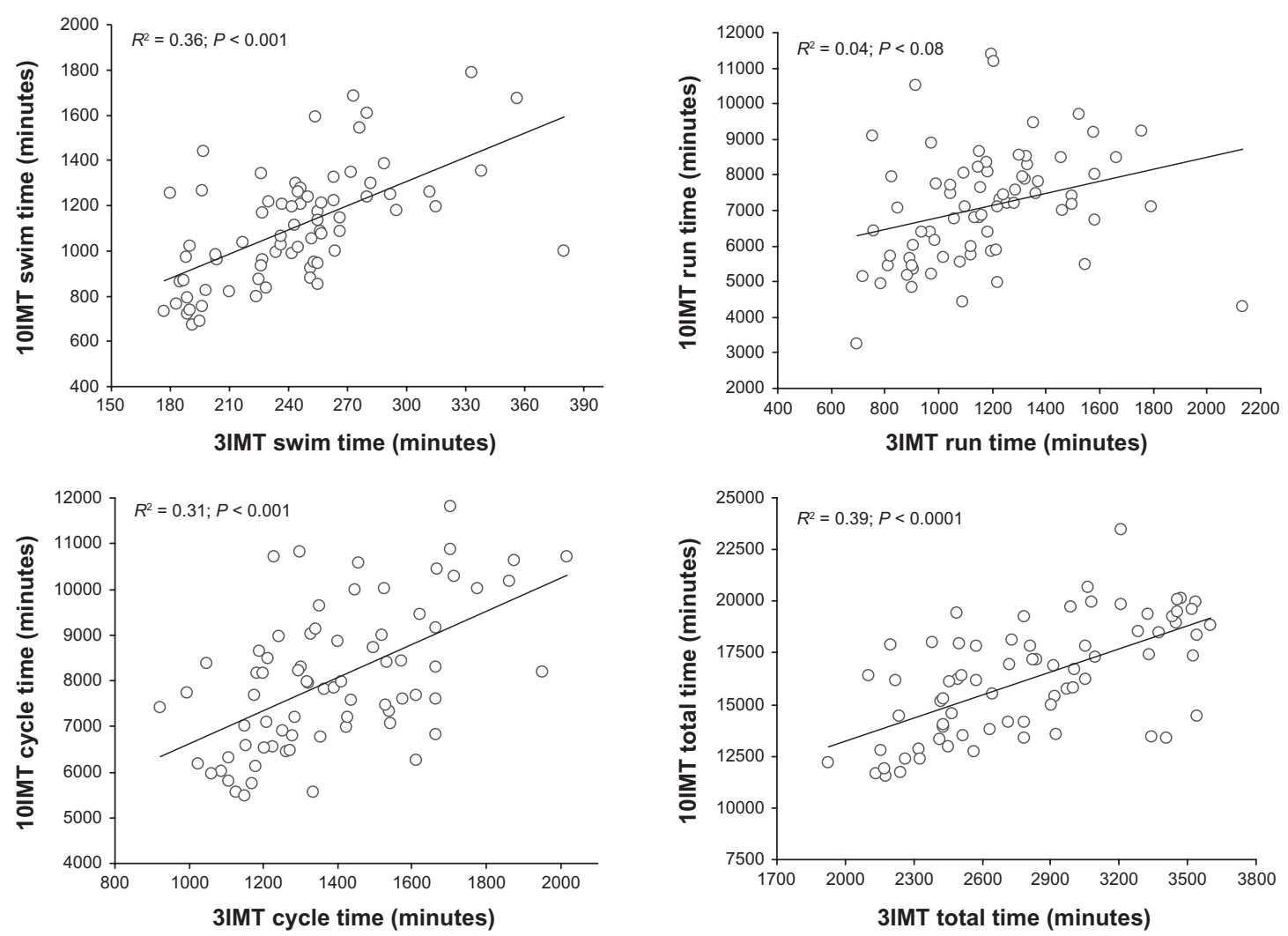

Figure 3 Relationships between triple (3IMT) and deca (IOIMT) Ironman distance triathlon performances for swimming, cycling, running, and total event.

part (less than $7 \%-9 \%$ of the total time) compared with the cycling (approximately 50\%) and running (approximately $41 \%-44 \%$ ) parts of ultra-triathlons. By comparison, for the Ironman triathlon, the $3.8-\mathrm{km}$ swimming, $180-\mathrm{km}$ cycling, and $42-\mathrm{km}$ running represent on average $11 \%, 52 \%$, and $35 \%$ of the total time, respectively. ${ }^{2}$ For 2 IMT, cycling and running performances are of the same importance in overall performance, while for 3IMT, running time seems to be a more important factor than cycling time to overall performance. These findings on 3IMT confirm the data from Knechtle and $\mathrm{Kohler}^{8}$ who found a significant correlation between total race time and both cycling time $\left(r^{2}=0.62\right)$ and running time $\left(r^{2}=0.87\right)$ but no correlation between swimming time and total race time in 17 subjects. Based on the observation that running performance was associated with race success in 3IMT, it could be hypothesized that the relative contribution of running performance to overall performance would increase with longer ultra-triathlon distance such 10IMT. However, we found that for 10IMT, running time was less related to total race time compared with cycling time. In addition, in contrast to swimming and cycling, 10IMT running performance could not be predicted based on 2IMT or 3IMT running performances. That finding could be explained in part by larger individual differences in running time compared with cycling time, probably related to fatigue and exhaustion during the run. In contrast to cycling, running performances in both 2IMT and 3IMT were not correlated to running performance in 10IMT; suggesting that factors associated with the performance of a 420-km run might differ from those associated with the performance on $126-\mathrm{km}$ run.

Numerous physiological factors (eg, muscle fatigue) and psychological aspects such as mental fatigue and loss of motivation may limit performance in the ultra-triathlon. Prolonged exercise inevitably leads to muscle fatigue. ${ }^{12-14}$ Central and peripheral mechanisms affect neuromuscular function during ultra-endurance exercise such as cycling and running. Recent studies suggest that central fatigue is the principal explanation for neuromuscular fatigue during prolonged exercise and that this minimizes the extent of peripheral fatigue. ${ }^{13}$ It would be interesting in the future to analyze muscle fatigue following ultra-triathlons to see how it differs from other ultra-endurance events such as prolonged cycling or running. ${ }^{12-14}$ Training in the three disciplines to prepare for the ultra-triathlon inevitably leads triathletes to perform a high training volume. For example, Knechtle et al ${ }^{7}$ showed that the average training volume of ultra-triathletes in preparation for a 3IMT corresponded to approximately 20 hours per week (3.5 hours swim, 10.2 hours cycle, and 
5.7 hours run). However, these authors found no correlations between training volume and 3IMT performance. More investigations are required on training characteristics of the ultra-triathletes, to gain a better understanding of how they prepare for such extreme challenges.

\section{Methodological considerations}

A limitation of the present study was that only performance times, age, and nationality of the subjects were available. It was not possible to collect other parameters related to performance such as physical characteristics, training volume, anthropometric parameters, or previous experience. ${ }^{6-9}$ Thus these factors had to be ignored in this study. Moreover, we have not considered environmental conditions, although research has shown that ultra-performances progressively slow down when the ambient temperature increases. ${ }^{15}$ In addition, the performance times in each discipline include all the time breaks necessary for feeding, massage, or sleeping. Because the timing clock is not stopped during the break, the average speed of each discipline did not correspond to the effective speed attained during the race. That may explain, in part, the very low cycling and running speeds observed for 10IMT, where athletes need to stop for sleeping. In further studies it would be interesting to analyze how 10IMT finishers manage the exercising time and rest time during a competition lasting approximately 11 to 12 days.

\section{Conclusion}

This paper is the first to fully describe ultra-triathlon performance. Results showed that swimming, cycling, and total performances were significantly correlated between the shortest ultra-distance triathlons (2IM and 3IMI) and the longest one, ie, 10IMT. In contrast, running performances in either 2IMT or 3IMT were not associated with 10IMT running performance. Running performance may be an important factor in 2IMT and 3IMT success; when distance increases to 10IMT, cycling performance seems to be predominant for success. For future ultra-triathletes who have already performed a 3IMT, and who intend to start a 10IMT, race time might be predicted by the following equation: 10IMT race time $($ minutes $)=5885+3.69 \times 3$ IMT race time (minutes).
Additional studies are required to determine the physiological and psychological factors associated with ultra-triathlon performance. We hope this paper will stimulate further research on the physiological profile of ultra-triathletes and the physiological demands of ultra-triathlons.

\section{Disclosure}

The authors declare no conflicts of interest in this work.

\section{References}

1. Knechtle B, Knechtle P, Lepers R. Participation and performance trends in ultra-triathlons from 1985 to 2009. Scand J Med Sci Sports. 2010. doi: $10.1111 /$ j.1600-0838.2010.01160.x

2. Lepers R. Analysis of Hawaii ironman performances in elite triathletes from 1981 to 2007. Med Sci Sports Exerc. 2008;10:1828-1834.

3. Lepers R, Sultana F, Bernard T, Hausswirth C, Brisswalter J. Age-related changes in triathlon performances. Int J Sports Med. 2010;31:251-256.

4. Lepers R, Maffiuletti N. Age and gender interactions in ultraendurance performance: insight from triathlon. Med Sci Sports Exerc. 2011;43:134-139.

5. Gastmann U, Dimeo F, Huonker M, et al. Ultra-triathlon-related bloodchemical and endocrinological responses in nine athletes. $J$ Sports Med Phys Fitness. 1998;38:18-23.

6. Lehmann M, Huonker M, Dimeo F, et al. Serum amino acid concentrations in nine athletes before and after the 1993 Colmar ultra triathlon. Int J Sports Med. 1995;16:155-159.

7. Knechtle B, Knechtle P, Rosemann T. Skin-fold thickness and training volume in ultra-triathletes. Int J Sports Med. 2009;30:343-347.

8. Knechtle B, Kohler G. Running performance not anthropometric factors is associated with race success in a Triple Iron Triathlon. Br J Sports Med. 2009;43:437-441.

9. Knechtle B, Knechtle P, Andonie JL, Kohler G. Influence of anthropometry on race performance in extreme endurance triathletes: World Challenge Deca Iron Triathlon 2006. Br J Sports Med. 2007;41:644-648.

10. Hoffman MD, Wegelin JA. The Western States 100-Mile Endurance Run: participation and performance trends. Med Sci Sports Exerc. 2009;41:2191-2198.

11. Hoffman MD. Performance trends in $161-\mathrm{km}$ ultramarathons. Int $J$ Sports Med. 2010;31:31-37.

12. Lepers R, Maffiuletti NA, Rochette L, Brugniaux J, Millet GY. Neuromuscular fatigue during a long-duration cycling exercise. J Appl Physiol. 2002;92:1487-1493.

13. Martin V, Kerhervé H, Messonnier LA, et al. Central and peripheral contributions to neuromuscular fatigue induced by a $24-\mathrm{h}$ treadmill run. J Appl Physiol. 2010;108:1224-1233.

14. Millet GY, Lepers R. Alterations of neuromuscular function after prolonged running, cycling and skiing exercises. Sports Med. 2004;34:105-116.

15. Wegelin JA, Hoffman MD. Variables associated with odds of finishing and finish time in a 161-km ultramarathon. Eur J Appl Physiol. 2011; 111:145-153.
Open Access Journal of Sports Medicine

\section{Publish your work in this journal}

Open Access Journal of Sports Medicine is an international, peer-reviewed, open access journal publishing original research, reports, reviews and commentaries on all areas of sports medicine. The manuscript management system is completely online and includes a very quick and fair peer-review system.

\section{Dovepress}

Visit http://www.dovepress.com/testimonials.php to read real quotes from published authors. 